\title{
Comparative Review of BCl Interface for Cognitive Performance
}

\author{
Rebakah Job \\ Asst.Professor \\ Kadi Sarva Vishwavidyalaya, \\ Gandhinagar, Gujarat, India
}

\author{
Ashish Jani, PhD \\ Associate Professor \\ P.P Savani university \\ Surat, Gujarat,India
}

\author{
Rupesh B. Vyas \\ Asst.Professor \\ Kadi Sarva Vishwavidyalaya, \\ Gandhinagar, Gujarat, India
}

\begin{abstract}
Brain Computer Interface is an approach through which people can interact with machines independent of muscles or nerve. Brain Computer Interface derives the electrical signals from the brain and converts the recorded analog signals into corresponding control signals with the help of functional computer. Brain Computer Interface is used to enhance the cognitive performance of a learner. Primarily, the review endows the acquisition of signal processing; there are differentiated activity functions of the brain in the form of electrical signals and magnetic signals in proportional to metabolic activity occurred. Secondly, the EEG signals control user intentions detected in brain activity. Thirdly, an overview of various BCI applications to classify the attention levels of the learners and help provided the enhancement in cognitive performance
\end{abstract}

\section{Keywords}

Brain Computer Interface, Cognition, Human -Machine interaction, Attention, EEG signals.

\section{INTRODUCTION}

Natural form of conversation involves muscles or nerves to convey, exchange, share ideas and feelings with a human intent. This process prompts a complex process in some of the brain areas. An indigenous current is engendered when neurons are elicited. EEG is the amount of signals that are generated during synaptic movement of the dendrites in the cerebral cortex of the brain[1]. Variances of electrical potentials are initiated by summation of postsynaptic graded potentials from pyramidal cells that create electrical dipoles between soma (body of neuron) and apical dendrites (neural branches). The membrane potential directed in the direction and inflated through channels entails of mostly sodium( $\mathrm{Na}+)$, potassium $(\mathrm{K}+)$, calcium( $\mathrm{Ca}++)$, and $\mathrm{Cl}$ - ions.[2]. The BCI machine executing Signal Processing and Pattern recognition deduce the signal activity occurring from the brain are denoted to a brain machine interface (BMI). It is a communication scheme expediting individuals to connect with their environments in the course of the segment where the operational of peripheral nerves and muscles does not transpire. BCI constructs a different strategy of relaying a person's intentions to a peripheral device such as Computers, synthesizers, assistive appliances by accessing the nonmuscular channel for relaying a person's intentions.[3]. There are five successive phases: signal acquisition, pre-processing, feature extraction, signal classification, and the control software after brain signals are placid from BCI over machine learning techniques. While the signals are procured, the first phase apprehends the brain signals and implements noise reduction program through artifact removal technique. This pre-processing phase assists to articulate the signals for supplementary process. The feature extraction phase regulates discriminative information in the brain signals that have been procured. These observed signals are drawn into vector enclosing operational and discriminant features. The abstraction of this stimulating data is a thought-provoking assignment. Brain signals are combined with other signals procured from a restricted set of brain activities that overlay in both time and space. Furthermore, the signals are not frequently fixed and are biased by artifacts such as electromyography (EMG) or electrooculography (EOG).The feature vector must also be of a truncated aspect, in order to shrivel the complexity of feature extraction phase, but without any loss of relevant information. The classification phase categorizes the signals captivating the feature vectors into different stages. The optimal discrimination features is therefore indispensable to accomplish operative pattern recognition, in order to decrypt the user's intents. Lastly, the control interface phase deciphers the classified signals into significant instructions sent to any connected device, such as a wheelchair or a computer.A substantial cognitive performance is accomplished when these signals are inferred and studied to determine learner attention levels as concentrated, attentive or relaxed.

\section{BACKGROUND}

The evolution of BCI has started in 1924. When the first examination was done on animals. Electroencephalograms (EEGs) are techniques which run an electrical depiction of bio-signal that reveal alterations occurring in the human brain activity. The basic requirement of these techniques and their expansion which has taken place in the last century give way to the synopsis of the pertinent programs and algorithms used to study EEG signals. The utilization of Brain Computer Interfaces outdoor to the laboratory has not been effectively carried out due to its robustness. Often signal processing algorithms provide weak performances while analysing the EEG signals.[4] the electric commotion befalling in brain is measured through a technique called as Electroencephalography (EEG). In the year 1875, the occurrence of flow of charge in form of electrical current taking place in the brain was discovered by English physician Richard Caton [5].This reflection of charge flow was detected in a unremitting routine and the same impulsive activity from the brain surfaces of animals like rabbits and monkeys. In the year 1912, Vladimir Vladimirovich Pravdich-Neminsky, an Russian physiologist published his work mentioning the discovery of the leading brain signals and the induced potentials in mammals $(\operatorname{dog})[5]$.As specified by Fuller that the German neurologist Hans Berger documented the occurrence of first human brain signal in 1924,which was the start of introducing that electrical signals were existing and can be digitally recorded [6]. A radio instrument is used to strengthen the mind electrical activity and the documentation was done on graph paper, as the device was entitled EEG.The 
periodic fluctuations in the brain fluctuates with the state of cognizance of the individual in accordance with the statement of Berger Franklin Offer, EEG equipment was developed and presented with concentric needle electrodes[7]. The occurrence of brain signals is combined with other unwanted signals which need methods of, detection, purification, classification of these signals, many researchers have worked on it. EEG topography was developed by English physician William Grey Walter for plotting of electrical activity occurring across the apparent base of the brain, used in psychiatry until the 1980s. During the era of 1990 to 2000 advances have been taken place in the technique to process brain signal Blind Source Separations (BSS) [8] and Independent Component Analysis ICA[9].BCI research is a bourgeoning across many platforms incorporating researchers from cognitive sciences, psychology, engineering streams like computer science, and health-care streams. In spite of some notable advances, a fundamental communication base has yet to be technologically advanced and its association along with the existing BCI technologies had progressively lessen the speed of research. A general feedback framework has to be established for the community of BCI researchers [10]. BCIs observe brain signals to obtain statistics on user's conation. BCI measure the brain activity and extracts the information into controllable electrical signals. Two types of brain activities that can be examined are Electrophysiological and Hemodynamic. Electrophysiological activity is engendered by electro-chemical transmitters that exchange information between the neurons. The neurons fetch about ionic currents which drift within and across neuronal associations. These intracellular currents are termed as primary currents. Preservation of electric charges mechanism enlarges that the primary currents are restricted by extracellular current flows, known as secondary currents The hemodynamic reaction is a procedure in which the blood discharges glucose to active neurons at a grander rate than in the expanse of inactive neurons[11]. According to the frequency of the signal, EEG signals are classified into different types. The frequency dimensions have been defined according to circulation of neurons over the scalp shown in Table 1 . The frequency recurs and classified as bands termed as delta $(\delta)$, theta $(\theta)$, alpha $(\alpha)$, beta $(\beta)$, and gamma $(\gamma)$ from low to high, respectively.

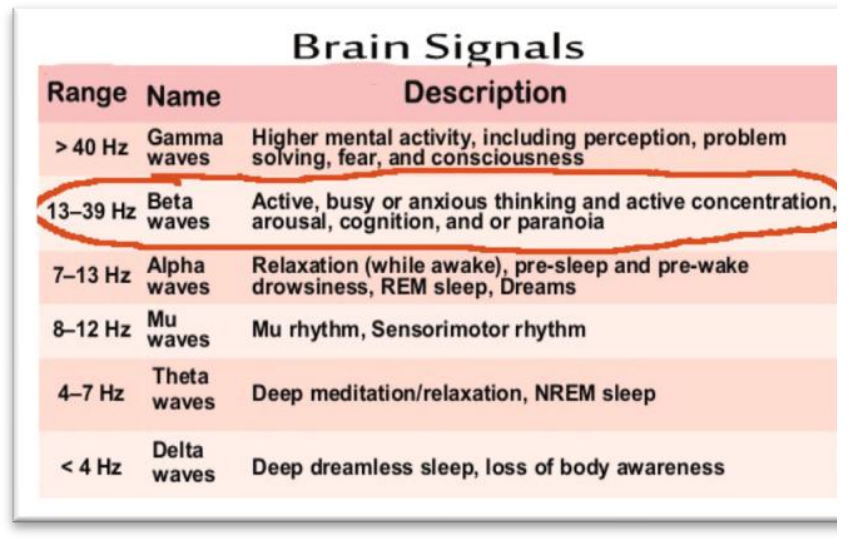

Figure 1: Description of Brain Signals

At the essence of all our thought process, sensations and performances is the passing of flow of charge between the neurons within the brain. Brainwaves are formed by harmonised electrical rhythms from the many types of neurons passing information between each other.The delta band frequency records below $4 \mathrm{~Hz}$ are observed in children below the age of one year and decline as they grow. In grown up individuals, Delta rhythms are customarily known in deep dreamless sleep and trifling in adults when awake. If these bands are recorded in colossal number signifying deformity and is linked to neurological disorder. Sometimes these bands are taken as artifact, which is a noise signal and caused by muscular movement of the neck or jaw. Theta band frequency ranges between 4 to $7 \mathrm{~Hz}$. An insignificant quantity of theta waves is chronicled if an individual is receptive and energetic. When the individuals are in drowsy or meditative theta waves are recorded at upper level. Comparable to delta waves, a huge amount of theta activity in receptive individuals is associated with neurological disorder. Theta band has been concomitant with meditation or concentration and a wide series of cognitive processes such as reasoning, or sensible state[11]. Alpha band frequency recorded between 8 to $12 \mathrm{~Hz}$. Their magnitude upsurges when individual eyes shut and the body relaxes and they incapacitate when the eyes are opened and mental thinking is on. Alpha activity is associated with logical reasoning. Cumulative mental effort and reasoning causes a conquest of alpha activity, particularly from the frontal areas[12]. Subsequently, these rhythms might be applicable signals to measure the cognitive skills. Beta band frequency ranges between 13 to $30 \mathrm{~Hz}[13]$ related with wakening mindfulness or responsiveness [13].Attention or concentration is measured when the brain signals are detected with beta waves. Beta brainwaves are classified into three bands depending upon the frequency; Low Beta $(12-15 \mathrm{~Hz})$ are associated with 'fast idle', or absorbed thought process. Beta $(15-22 \mathrm{~Hz})$ is associated with logical thinking or dynamically problem solving ability. High Beta $(22-38 \mathrm{~Hz})$ is associated with intricate thinking ability, incorporating new capabilities, high apprehension. Persistent frequency of brain signal processing is not a proficient way to invoke the brain, as it takes a remarkable energy.Gamma band frequency signals are recorded from the range of 30 to $100 \mathrm{~Hz}$. The existence of gamma waves in the brain activity of a healthy individual is connected to certain muscular movements. Certain research trials have publicized an association in individuals between motor actions and gamma waves have been traced during muscle contraction [3]. The EEG signal recording system consisting of electrodes with conductive media, amplifiers with filters, A/D converter and recording device. To detect a $\mathrm{BCI}$ readings, electrodes are used and they are basically there are basically of the following types :one-use (gel based), reusable electrodes (gold-plated, silver-plated, stainless steel or tin), headset and EEG cap, electrodes using saline-based ,needle electrodes [9]. There are some companies' hosted BCI games in the market. Company named Emotiv[14] has by now technologically advanced and introduced several set of BCIbased games, such as Cortex Arcade and Spirit Mountain Demo Game. Besides, the company owns a high efficient BCI with 14 electrodes at very reasonable price called as EPOC Neuro Headset (Figure 1 (b)) along with a friendly interface for programmers. This set a fast development strategy to the BCI-based applications. NeuroSky, another company also arcades the a headset named MindWave Neuroheadset [14] (Figure 1(a)) with inbuilt interface for developing software applications that can accumulate brainwaves and derivate into various mental states. Many other software companies like Microsoft have revealed curiosity to conduct research on BCI, inquisitive of the expansion of preliminary original applications that used Brain Computer Interface. The MindWave Mobile Headset consist of the measuring device, sensor that contacts with the forehead, and orientation points are positioned on the ear pad, and the micro-controller chip processes the signals and delivers this data to applications in 
digital form. This instrument is connected to the analyser through Bluetooth connectivity. The EEG electrode is positioned on the individual forehead (on the frontal cortex) during the course of experiment. The headset securely processes the signals and delivers outputs in form of EEG power scales (alpha waves, beta waves, etc), which rate to the level of attention, meditation and measure of eye blink. Ranges of Attention and Meditation are specified and conveyed to a meter with a virtual eSense range of 1 to 100 . Values between 20-40 are abridged range which are not suitable for consideration and from 1-20 they are reflected strongly sunk eSense values. the value falling in the range of $40-60$ is reflected to be unbiased. The resulting Values greater than 60 are considered to be elevated values". Values in the range of 80-100 are considered to be high levels of eSense.

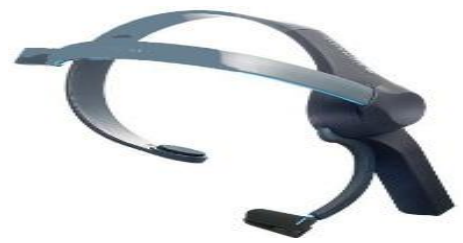

Figure 1(a) Mind Wave Mobile

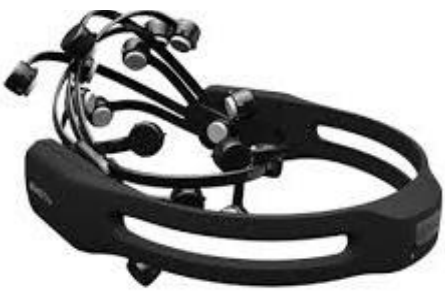

Figure 1(b). EmotivHeadset

\section{BCI APPLICATION IN EDUCATION AND CHALLENGES}

Brain Computer Interfaces have thriving applications in numerous arenas of research. Divergent practices are used to make the of BCI utilization in assessing attention levels.

\subsection{Monitoring through Camera}

Brain Computer Interface (BCI) is a technique of interacting with a peripheral device grounded to detect and measure signals derived from the brain. A Specific brain signals named the P300 potential, are detected about $0.3 \mathrm{sec}$ after an intentional cognitive envelopment to outward incitement. P300 utilization has led to advancement in designing BCIsupported training tool directing to improvement of attention. An efficient economical camera using infrared rays working with an algorithm called as "off-axis perspective projection" to achieve the depth in the degree of artifice in 3D environment to engross the students. The experiment regulates measurement collected from a particular electrode located on the scalp above the parietal lobe (Pz).To evoke $\mathrm{P} 300$ potential two arrangements of trials have been executed. One of the trial was operated the features of "P300 speller" and the second system applied in an environment where the user prerequisite was to explore a particular letter in a sessions of altering graphic images[15]. The compilation of results and feedback are accumulated from the healthy individuals who contributed in participating in the P300 based Virtual Reality system. This experiment proved for drug free treatment for ADHD subjects. The programmed P300 detection classifier algorithm was accomplished through sequence selection of factors derivate from a prototype of the P300 signal. Support Vector Machine classifier has been used to detect P300 potential. Individuals are availed to recognise the overriding colour more accurately than a unique colour. The observation states that dawdling feedback is recorded than usual Neuro feedback practice and this huge variance portrays an ample quantity of data.

\subsection{Monitoring using Augmented Reality}

Augmented Reality is a technique used for virtual visualization over the real world aspects. To determine the spatial memory a research using Augmented Reality (AR) is carried out - ARSM (Augmented Reality Spatial Memory) task. Spatial memory is responsible memory for recording the surrounding environment. The challenge was to demonstrate the effect of the augmented reality in evaluating spatial memory with the associated subjects [17]. The technique of implementing ARSM task was conducted to evaluate the individual skill to remember visual information in the surroundings. It has high credibility to access spatial shortterm memory skills in real world and evaluated the ARSM task's reusability and apparent approval. The ARSM task is developed using Unity and vuforia, code developed in C\# and 3D models are designed in Autodesk 3DStudio. The results are assessed using ANOVA test determining the suitable device size used for ARSM task. The tasks evaluated the capability of a subject to recognize and remember the cumulative locations of objects that appeared in AR. Comparing the ARSM task's performance with traditional neuropsychological procedures with respect to the user friendliness and fulfillment of the participants for the ARSM task. The enactment of the ARSM task exhibited improvement in the short-term memory of age 5-8 year's children. This task can be recommended to be used as an amusing method to evaluate or provide training to subjects children in spatial short-term memory services. Further study needs to be conducted to provide more accuracy to the above statement, to enhance the retention of memory and identify academic challenges with individuals having with academic complications or neurological disorders.

\subsection{Monitoring in Classroom}

A system is developed to monitor attention in the classroom during the phase of lecture conducting and identify the concentration to the lecturer drops in concentration. This system is developed considering four phases: Capture, Report, and Predict, Act and Refine to access Classroom Attention by Mirko Raka et all [19]. An infra-red camera is installed in the classroom when the lecture session is in process; the data is captured as the prime data through videos captured from the classrooms, which is acquiescent in influential a minimum impact on the existing classroom environment. The passive way to capture the images from the classroom is done by installing observational cameras with no expectation of interaction from subjects' side. The introductory phase of the experiment involves challenging the various visual reference markers to measure attention during the lecture sessions, presently giving priority on two features - quantifying body motion and estimation of a gaze direction. The two phases in the experiment emphasis on "Reaction on Action", by awarding a abstract study of the attention gained in the classrooms to the teacher. The third Act phase is the notion is that the students in the class are paying attention will have greater harmonization responding in actions reflecting a higher possibility of jotting down information in response to the session they are hearing. The next phase is Refinement with respect to the teacher to prepare the lessons before taking the sessions as it is in the experimental segment of the task, the statistics and technologies will pass into numerous cycles 
of iterations before the conclusion of their significance in the final framework. The challenges faced in the time of enormous manipulation in virtual learning environments of new concepts, an improvement is needed for typical learning situation. The advanced technologies of visual programming are enabled to afford appropriate statistical study of the present learning environment in an unremarkable way, and the teachers are enhanced with diversified tools to deliver better session.

\subsection{Monitoring through Mind Builder}

Some major software companies like EMOTIV and Neuro Sky have developed efficient economical Headsets to measure the brain waves so that Attention can be measured not based on external factors. NeuroSky Mind Builder-(MB) device is considered as a device utilized for measuring attention[16]. A characteristic of attentiveness was to investigate whether the mind mobile readings can be collective generated along with user data. The consolidation of biological and user- generated data would permit the advance programming sessions of more refined models. A model named Second life is generated to assess Mind Wave mobile usability. When the learner assess data, the brain waves are generated and following assessment exercise was developed to measure the knowledge gained during the teaching phase. The exercise holds in arrangement in inclination with a model of attention[17] developed around self-motivated variables detected in the learner's brain which are included as MB inputs and the learner's activities in a computer-based knowledge domain. The preparation, nevertheless, blends Mind Mobile readings which provide a more precise evaluation of the learner's attention based on brain activity with data produced by the user. Accordingly the prototype of the attention measurements are calculated based on the information collected from the score derived from assessment test or the time taken to answer each question for standard attention. The MB reads attention levels in an random scale ranging values from 0 to 100 . There is an preliminary interval of time gap between 7 and 10 seconds for the headset to be mounted and configured to detect the brain signals. The attention measurement is calculated after the values are derived from the headset and converted to digital form at a rate of 1 Hertz. A negative value is designated when there is no signal is being detected from the headset and the moment signal starts accelerating the values are transferred to the interface and the range lies between 0-100. The acquired dynamic signals from the brain detection instrument depicts the pattern of attention detected in the course of experiment and the acquired data sets indicates the model of attention supporting the assessment exercise associated to accurate learning session for a specific duration of time.

\section{EXPERIMENTAL ANALYSIS}

The comparison review of the four methodologies used to monitor Attention during a span of time and each experiment is carried out using hardware devices like camera, mobile device, Neuro Headset and the inputs are further analyzed and processed to attain the results. Traditional methods to test the attention gained use survey methods, questionnaire which are data collected voluntarily, but if we want to know the real time brain signals at the time of learning process, the approach of collecting data from BCI supports in the real time environment. The above discussed four different experimental settings have been conducted with the diversified population ranging from school children to graduate.

\section{RESULTS}

The BCI Application in education, the experimental analysis has been carried and the results obtained with respect to the factors are in comparison denoting \#1- Monitoring through Camera, \#2-Monitoring using Augmented Reality, \#3Monitoring in Classroom Attention, \#4-Monitoring through Mind Builder. 12 factors are considered and compared with respect to the methodology adapted. Each methodology has attained different results in concurrence with the environment.

\begin{tabular}{|c|c|c|c|c|}
\hline Factors & $\# 1$ & $\# 2$ & \#3 & \#4 \\
\hline Hardware & $\begin{array}{l}\text { Infra-Red } \\
\text { Camera }\end{array}$ & $\begin{array}{l}\text { Motorola } \\
\text { Xoom2, } \\
\text { 3D printer }\end{array}$ & $\begin{array}{l}\text { Video } \\
\text { Camera }\end{array}$ & $\begin{array}{l}\text { Mind } \\
\text { Wave }\end{array}$ \\
\hline Algorithm & $\begin{array}{l}\text { Off Axis } \\
\text { projection }\end{array}$ & ARSM & $\begin{array}{l}\text { Annotate } \\
\text { person } \\
\text { location }\end{array}$ & $\begin{array}{l}\text { Propri } \\
\text { etary } \\
\text { algorit } \\
\mathrm{hm}\end{array}$ \\
\hline $\begin{array}{l}\text { Brain } \\
\text { Signal }\end{array}$ & P300 & NA & NA & $\begin{array}{l}\text { Beta } \\
\text { wave }\end{array}$ \\
\hline Signal Rate & $256 \mathrm{~Hz}$ & NA & NA & 100 \\
\hline $\begin{array}{l}\text { Displayed } \\
\text { Stimulus }\end{array}$ & $700 \mathrm{~ms}$ & $\begin{array}{l}200-500 \\
\mathrm{~ms}\end{array}$ & offline & $\begin{array}{l}100 \\
\mathrm{~ms}\end{array}$ \\
\hline $\begin{array}{l}\text { No of } \\
\text { Electrode }\end{array}$ & $\begin{array}{l}\text { Single } \\
\text { over } \\
\text { parietal } \\
\text { lobe } \\
(\mathrm{Pz})\end{array}$ & NA & NA & 2 \\
\hline $\begin{array}{l}\text { No of } \\
\text { temporal }\end{array}$ & 0.4 & 0.6 & 0.5 & 0.3 \\
\hline $\begin{array}{l}\text { Target } \\
\text { Audience }\end{array}$ & ADHD & $\begin{array}{l}\text { pre-school } \\
\& \\
\text { Primary } \\
\text { School }\end{array}$ & UG & UG \\
\hline $\begin{array}{l}\text { Capturing } \\
\text { Method }\end{array}$ & camera & $\begin{array}{l}\text { Augmente } \\
\text { d Reality }\end{array}$ & $\begin{array}{l}\text { body } \\
\text { motion, } \\
\text { gaze }\end{array}$ & $\begin{array}{l}\text { single } \\
\text { sensor }\end{array}$ \\
\hline $\begin{array}{l}\text { Duration of } \\
\text { experiment }\end{array}$ & $\begin{array}{l}45 \\
\text { minutes }\end{array}$ & 45 Min & 45 Min & $\begin{array}{l}2 \\
\text { Min }\end{array}$ \\
\hline $\begin{array}{l}\text { Software } \\
\text { used }\end{array}$ & SVM & $\begin{array}{l}\text { Unity } \\
3 \mathrm{D}, \mathrm{C} \#, \mathrm{Vu} \\
\text { foria }\end{array}$ & $\begin{array}{l}\text { Machine } \\
\text { learning } \\
\text { technique }\end{array}$ & $\begin{array}{l}\text { AI, } \\
\text { C\# }\end{array}$ \\
\hline $\begin{array}{l}\text { No of } \\
\text { Questionnai } \\
\text { re }\end{array}$ & Nil & 6 & Nil & 7 \\
\hline
\end{tabular}

measures

\section{CONCLUSION}

$\mathrm{BCI}$ is the exploring way to enhance the cognitive performance of the learners. The future work include selfreported attention and self-reported motivation as a baseline variable to understand the learner capacity and the level of attention attained in the class room at a particular amount of time levels of attention. The intention of developing an attention model was to aid learners to increase or sustain higher attention levels to influence their motivation. Many 
traditional systems have acquired data based on questionnaire and survey to detect the attention span, system derivation images from the specific classroom and the body posture is studied to determine attention levels. Augmented Reality is used to depict some images to the learner for a flash of time and the user is expected to remember the positional change of the images or monitor the change with respect to colour, size of the image and have to answer likewise. The intervention of BCI has given the chance to go beyond the superficial environment to the real environment to collect the data from the brain waves and determine the actual attention span. The future model can be generated long with the signals derived from the brain waves and gaze estimation to derive the attention span. The study of brain signals in the real environment helps to enhance learning process in the special disabled children facing concentration problems. The BCI application in the classroom where the facilitator is aware of the real percentage of knowledge being absorbed can modify accordingly to the specific needs of the learner. The content can be iteratively modified till the maximum benefit is derived by the learners.

\section{REFERENCES}

[1] "4th Kuala Lumpur International Conference on Biomedical Engineering 2008," vol. 21. 2008.

[2] M. Teplan, "Fundamentals of EEG measurement," Meas. Sci. Rev., vol. 2, no. 2, pp. 1-11, 2002.

[3] L. F. Nicolas-Alonso and J. Gomez-Gil, "Brain computer interfaces, a review," Sensors, vol. 12, no. 2, pp. 1211-1279, 2012.

[4] C. Umale, "Feature Extraction Techniques and Classification Algorithms for EEG Signals to detect Human Stress - A Review," vol. 5, no. 1, pp. 8-14, 2016.

[5] A. Coenen and O. Zayachkivska, "Adolf Beck: A pioneer in electroencephalography in between Richard Caton and Hans Berger.," Adv. Cogn. Psychol., vol. 9, no. 4, pp. 216-21, 2013.

[6] B. R. Hackshaw, U. Sinclair, and R. E. G. Hackshaw, "Electrified Thoughts: the Early History of Eeg and American Pop Culture," vol. 16, no. 4, pp. 26-28, 2007.

[7] J. Sunday and N. Henry, "Brain Computer InterfaceAn Eye on Electroencephalogram ( EEG ) Applications in Clinical Medicine," vol. 3, no. 7, pp. 492-500, 2016.

[8] C. Brändström, "Using the Internet in Education Strengths and Weaknesses and Instruction," 2011.

[9] M. Ungureanu, C. Bigan, R. Strungaru, and V. Laarescu, "Independent Component Analysis Applied in Biomedical Signal Processing Politehnica," J. Inst. Meas. Sci. SAS, vol. 4, no. 2, pp. 1-8, 2004.

[10] L. M. S. Morillo1, J. A. Alvarez-Garcia1, L. Gonzalez-Abril, and J. A. O. Ramírez, "Discrete classification technique applied to TV advertisements liking recognition system based on low-cost EEG headsets," Biomed. Eng. (NY)., vol. 15, no. 1, pp. 198-218, 2016.

[11] M. J. Khan and K.-S. Hong, "Passive BCI based on drowsiness detection: an fNIRS study," Biomed. Opt.
Express, vol. 6, no. 10, p. 4063, 2015.

[12] W. Klimesch, "EEG alpha and theta oscillations reflect cognitive and memory performance: A review and analysis," Brain Res. Rev., vol. 29, no. 2-3, pp. 169-195, 1999.

[13] A. L. Crowell et al., "Oscillations in sensorimotor cortex in movement disorders: An electrocorticography study," Brain, vol. 135, no. 2, pp. 615-630, 2012.

[14] "Emotiv EPOC neuroheadset [5]; (b) Neurosky Mindwave _ Open-i.".

[15] D. A. Rohani and S. Puthusserypady, "BCI inside a virtual reality classroom: a potential training tool for attention," EPJ Nonlinear Biomed. Phys., vol. 3, no. 1, p. 12, 2015.

[16] G. Rebolledo-Méndez, S. De Freitas, J. R. RojanoCaceres, and A. R. Garcia-Gaona, "An empirical examination of the relation between attention and motivation in computer-based education: A modeling approach," Proc. 23rd Int. Florida Artif. Intell. Res. Soc. Conf. FLAIRS-23, no. Flairs, pp. 74-79, 2010.

[17] G. Rebolledo-mendez, I. Dunwell, and E. A. Martínez-mirón, “2009 Assessing NeuroSky's Usability to Detect Attention Levels in an Assessment Exercise.pdf," pp. 149-158. 